\title{
The Motif Tool Assessment Platform (MTAP) for Sequence-Based Transcription Factor Binding Site Prediction Tools
}

\author{
Daniel Quest \\ Oak Ridge National Laboratory \\ Biological Sciences Division, Computational Biology Group \\ Building 5700 \\ P.O. Box 2008, MS 6164 \\ Oak Ridge, TN 37831-6164 \\ questdj@ornl.gov
}

\author{
Hesham Ali \\ University of Nebraska \\ College of Information Science \& Technology \\ Department of Pathology and Microbiology \\ Omaha, NE 68182 \\ hesham@unomaha.edu
}

\begin{abstract}
Predicting transcription factor binding sites (TFBS) from sequence is one of the most challenging problems in computational biology. The development of (semi-)automated computer-assisted prediction methods are needed to find TFBS over an entire genome, which is a first step in reconstructing mechanisms that controls gene activity. Bioinformatics journals continue to publish diverse methods for predicting TFBS on a monthly basis. To help practitioners in deciding which method to use to predict for a particular TFBS, we provide a platform to assess the quality and applicability of the available methods. Assessment tools allow researchers to determine how methods can be expected to perform on specific organisms or on specific transcription factor families. This chapter introduces the TFBS detection problem and reviews current strategies for evaluating algorithm effectiveness. In this chapter, a novel and robust assessment tool, the Motif Tool Assessment Platform (MTAP) is introduced and discussed.
\end{abstract}

Key words: Transcription Factor Binding Sites (TFBS), prediction algorithms, assessment tools, Motif Tool Assessment Platform (MTAP).

\section{Introduction}

Transcription factors and other regulatory proteins bind to DNA primarily around the transcription start site, interact with RNA polymerase and then facilitate or inhibit transcription of the gene. Most transcription factors bind to DNA at sequence-specific positions along the chromosome, called transcription factor binding sites (TFBS). The (partially) conserved sequence pattern found at several sites bound by the same transcription factor is called a motif. Motifs co-occur near transcription start sites for genes that are 
regulated by the same transcription factor. Many computational approaches have been developed to find conserved motifs in the regulatory regions upstream of genes that have similar expression patterns. Computational approaches complement experimental approaches because they are less labor intensive and costly. In addition, a predictive computational model is very useful when experimental data is limited.

\section{1 . TFBS Detection Problem}

In prokaryotes, given a set of genes that are differentially expressed, i.e., partially controlled by the same set of transcription factors, the TFBS identification problem is to mark conserved patterns in the regulatory regions of the differentially expressed genes. The patterns can be represented as a set of $k$-mers (words of length $k$ ) or as a Position Specific Scoring Matrix (PSSM) among others. When the pattern is represented as a set of $k$-mers, the objective function to be minimized is the number of mismatches in the set of words such that there exists a binding site in close proximity to the transcription start site for each differentially expressed gene. When the pattern is represented as a PSSM, the objective is to maximize the probability that a PSSM of a given length co-occurs in the promoters of each of the differentially expressed genes (1).

Regardless of the approach taken to represent motifs at the binding sites, practitioners must balance a set of complex trade-offs when building tools to solve the TFBS detection problem. Hence, in the detection process, motif representation is the first step. After motifs are represented, all possible motif instances in the differentially expressed promoters are indexed. Then, a distance function is used to discriminate motif instances that exist in the promoters of the differentially expressed genes but do not exist in background sequence. Finally, likely matches are extracted, ranked, and reported.

Currently, there are almost 200 tools to find TFBS motifs given a set of differentially expressed genes. For the current list, refer to http: //biobase.ist.unomaha.edu/ mediawiki/index.php/Main_Page. For many practitioners, the most pressing question is 'what prediction tool should I use?' Experts in the field commonly recommend running a set of tools and manually comparing the outputs. This has some merit, but a more formal methodology is needed to rank tools for different problem characteristics.

\subsection{Algorithm Evaluation}

One way of choosing the most appropriate tool for a specific problem is to run each possible tool on a related problem where there is experimental evidence. The experimental evidence can then be used as a standard to measure tool predictive performance. Ideally, one would just run each tool as a black box to mark TFBS, and then compare the TFBS predictions with the known binding sites found in the database. The most appropriate tool for a problem is one that correctly predicts the largest percentage of known binding sites (true positive predictions) while at the same time marking the least amount of non-sites, regions that have similar sequence composition to known motifs but are not known to be bound by a transcription factor (false positive predictions).

Each prediction algorithm requires multiple and different stages in order to make a prediction. Each stage corresponds to a unique added value implemented by the method. Some methods implement novel approaches for modeling background sequences, other methods implement cross-species conservation models, while others include data from other sources such as expression arrays. Thus, diverse TFBS prediction algorithms cannot be treated as black boxes with the same input and outputs. Evaluation of several different TFBS detection methods requires that we build pipelines for all methods. These pipelines allow 
access to all of the same data sources and standardize the outputs so that they can be compared (Fig.??.1). Once the predictions are generated from all of the tools, statistics are collected that measure the number of overlaps found between predictions and known TFBS.

\section{Materials}

\subsection{The MTAP Software}

The software discussed in this Chapter, Motif Tool Assessment Platform (MTAP), was implemented in Python, $\mathrm{C} / \mathrm{C}++$, Java, and Perl. A large assortment of languages was used because many effective algorithm techniques from other authors are included in the MTAP download and are implemented in several different languages. MTAP is open source, free, and community supported. Enhancements are welcome. A community supported list of known TFBS finding algorithms can be viewed at http://biobase.ist.unomaha.edu/mediawiki/ index.php/Main_Page. MTAP and a User's Manual can also be downloaded from this site. The installation of MTAP, due to its complex dependencies, is far from being trivial as described in Note 1. For running MTAP, see Note 2.

\section{Methods}

\subsection{Algorithms}

The central challenge in evaluating how well tools predict TFBS is collecting datasets that in some way constitute a meaningful representation of a (small) part of the transcription regulatory networks. It is likely that the large number of prediction tools exist primarily because the problem is difficult to pose. Despite this, computational predictions have proven useful in narrowing the search space for many known TFBS. Recently, databases have been developed that contain binding site information for a large number of transcription factors. High-throughput sequencing technologies and high-density micro-array based technologies enable the construction of such TFBS databases. One application of these databases is to use it as a source of comparison with prediction algorithms, which should enable refinement of the tools and models used for TFBS prediction. The ability to accurately evaluate how well TFBS prediction algorithms correspond to TFBS databases is critical to understanding the faults of current methods and possible avenues for improvement.

\subsection{TFBS Databases}

As experimental evidence mounts, TFBS locations have been collected and entered into regulatory databases. Significant progress has been made identifying regulatory genes, signaling pathways, and transcription factor binding sites. Pathways responsible for a wide variety of cellular processes have been identified in Escherichia coli, Bacillus subtilis, yeast, worms, fruit flies, sea urchins, zebrafish, frogs, chicken, mice, and humans, just to name a few. The most substantial progress in constructing multi-cellular organism regulatory maps has been with the sea urchin embryo (2), the characterization of the dorsal-ventral patterning of early Drosophila embryo (3) and the detailed map of Ciona intestinalis (4).

The E. coli regulatory map (5) has been built by combining the work of Shen-Orr et al. (6), the curators of RegulonDB (7) and the maintainers of EcoCyc (8). This unique annotation contains a large network topology representing current understanding of gene regulation in $E$. coli K12. The annotation includes binding positions derived from experimental evidence for E. coli K12 regulatory proteins. Transfac (9), DBTBS (10), RegTransBase (11), and Prodoric (12) are all examples of TFBS databases that have been developed in recent years to annotate the regulatory network in other organisms. 
A standard for annotating binding sites is still emerging. In most databases, a TFBS is annotated in a database with a start position, end position, and strand information. Some databases contain additional information such as the genes regulated, the protein family of the transcription factor and the type of regulation (e.g., activation or repression). The information found in these databases has not been standardized. Consequently, many useful properties such as the strength of the interaction between the transcription factor and the binding site are not available. Some databases differentiate between DNA regions that are bound by transcription factor and those that lie between interacting sites. Despite the need for protein-DNA interaction information in protein structure data, few databases incorporate structure data in the annotation. The structure and representation of TFBS information in the database limits the accuracy of TFBS detection. Many researchers currently believe that even the most comprehensive databases miss many sites, especially those with weak interactions. Some researchers build synthetic datasets to circumvent these issues, but these approaches are limited by the level of fidelity of synthetic test representing the biology of transcription factor binding. Appropriate evaluation metrics are essential for determining the type and structure of data that should be cataloged to improve TFBS prediction.

\subsection{Core Evaluation Statistics}

Algorithm performance is evaluated by comparing the positions of predicted sites to the positions of known sites. For each position marked, seven core statistics are collected. The first four core statistics, shown in Table ??.1 are $n T P$-nucleotide true positives, $n F N$ nucleotide false negatives, $n F P$-nucleotide false positives, and $n T N$-nucleotide true negatives. They are collected by adding the number of each occurrence for each position in the regulatory regions.

The site level statistics ( $s T P$-site true positives, $s F N$ - site false negatives, and $s F P$-site false positives) are the final three core statistics. A site level statistic encompasses the idea that a group of adjacent nucleotides, marked as binding positions for a specific transcription factor, is representative of a binding site annotation. A site is a true positive if the prediction overlaps the annotation by no less than $\tau$ percent (a threshold) of the site. Site true negatives $(s T N)$ represent any collection of adjacent bases that are not predicted or annotated to be a site. The total number of such sites grows as a triangular number (13). However, once a site is annotated or predicted, all possible overlapping sites can no longer be marked a $s T N$. This makes $s T N$ less meaningful because it can increase or decrease depending on the number of predictions and annotations in the dataset. In practice, it is best to set this number sufficiently large so that it is always greater than $s T P, s F N$, and $s F P$ and always positive and consistent regardless of the number of predictions and annotations in the regulatory regions. Our convention sets this value to the length of all sequences in the upstream set, $n T P+n F N+$ $n F P+n T N$ divided by the number of sequences in the co-bound set. We then subtract the number of predictions and annotations from this total when calculating statistics. This ensures that $s T N$ will always be a strictly positive number that is independent of the number of predicted and annotated sites. The site level statistics are shown in Table ??.2.

When evaluating site level statistics, setting the value of the threshold $\tau$ is important. Tompa et al. (14) set $\tau$ to $25 \%$. Assuming this overlap, if an experimentalist were to remove the site, a change in expression should be observed. In some organisms, such as bacteria, this threshold is too strict because the width of known binding sites is too large for some tools to ever achieve a $s T P$. Many motif discovery programs have fixed motif widths (e.g., 8 base pairs), a threshold of $25 \%$ would not be sufficient to mark sTPs (e.g., an annotated site of width 60 and a site prediction of length 8). Site level motifs could be ranked based on a 
percentage of the prediction width instead of the motif width in the annotated database, but this would give an unfair advantage to methods that predict larger sites. In the example benchmarks in this chapter, $\tau$ is set equal to the maximum annotated site width in the dataset divided by the minimum expected motif width predicted by the suite of programs times $25 \%$. A degree of overlap indicates that computational and biological refinement of site predictions can still find the site. Table ??.3 illustrates the seven core statistics collected for algorithm evaluation.

For each transcription factor, a set of regulatory regions $n$ bases upstream of the controlled genes is collected, and each of the seven core statistics is collected for each of the upstream regions. Note that a given tool will be run for each set of co-bound regulatory regions separately but that the annotation is considered only once. For example, consider a set of regulatory regions bound by two transcription factors, $A$ and $B$. $A$ and $B$ cooperate in the same regulon to control a set of genes $X$ (those genes only controlled by $A$ ), $Y$ (those genes controlled by both $A$ and $B$ ), and $Z$ (those genes only controlled by $B$ ). Consider the set of regulatory regions collected to calculate the TFBS for $A$ (regulatory regions for $X$ and $Y$ ). Predictions from regulatory regions regulating $Y$ that overlap $B$ 's TFBS can be marked as false positives because they predict a TFBS other than the protein of interest.

The allowed prediction threshold indicates how many TFBS predictions are allowed by a tool. TFBS predictions come in sets and each set represents a highest scoring representative of binding sites for one transcription factor. In many cases, the highest scoring representative is just a sequence that happens to co-occur in the co-bound regulatory sequences and is not representative of a TFBS. For this reason, practitioners often accept more than one prediction. Allowing more predictions than one from a tool has the advantage that more true sites can be detected and tools can then better represent the combinatorial and co-operative regulatory cellular interactions that often occur. Varying the allowed prediction threshold has dramatic impacts on tool performance characteristics.

\subsection{Derived Evaluation Statistics}

More advanced metrics for performance evaluation can be calculated from the seven core statistics. Tompa et al. (14) recommended the six informative statistics shown in Table ??.3. Each of these statistics has its merit and is informative in different ways, depending on the objectives of the assessment. It is difficult to build tools that have high sensitivity and specificity. The Sensitivity/specificity tradeoff and the Matthews correlation coefficient, $n C C$, ( $n C C$ takes values -1 to 1 with 0 representing not correlated) are often viewed as an overarching measurement for performance.

There are two central problems in the TFBS databases used for evaluation. First, most datasets are incomplete, since many TFBS are not annotated in the dataset. The best way to avoid misleading scoring of a TFBS detection method is to construct a dataset that is as complete as possible to diminish the possibility of false positives. The second problem shows up when a method is over-fit to the known data repositories. Over-fitting occurs when the method training set and testing set are too similar. Because so little data has been available on TFBS, many methods have been optimized to find binding sites that have already been discovered. It is impossible to say how well tools will detect unknown binding sites in the future.

In the machine learning community, cross-validation is often used in supervised learning problems. Leave one out cross validation refers to training of an algorithm on a subset of the available data and testing on the subset of the data that is left out. Leave $n$ out crossvalidation refers to an iterative training and testing process where the data is partitioned into 
many sets. At a given stage in the cross validation process a subset of the data is either used for training or testing. All sets are eventually used for both training and testing. The benchmark of algorithm performance is constructed from combining the values from multiple benchmarks on each partition of the dataset dedicated to testing. In the context of new algorithm development, MTAP can be used in either of these ways. Historically, most algorithm developers did not view the TFBS detection problem as a supervised learning problem; instead it was viewed as an unsupervised learning problem. In other words, tool developers did not divide known TFBS instances into testing and training sets, actually, most often a training set did not exist. Instead, the goal was to build methods that could discover the first TFBS with the eventual goal of constructing large datasets for supervised learning. Most often, this was because of the lack of known TFBS. It is impossible to determine what TFBS influenced tool developers in the development process and should therefore be discarded in evaluation metrics. The most common goal of MTAP is to rank tools on a particular dataset given a recommended runtime procedure recommended by the tool author. The dataset used in this evaluation is assumed to be independent from the data used by the algorithm developer to construct the technique.

\subsection{Combining, Viewing, and Evaluating Datasets}

Once each tool is run over datasets $D=\left\{d_{1}, d_{2}, \ldots\right\}$, the results need to be illustrated in a meaningful way. There is an ongoing discussion on the best approach. Tompa et al. (14) proposed three methods for combining the results into one graph. Sandve et al. (15) proposed a method for evaluating results based on how well instances of the motif conform to known binding models. We proposed a method based on ROC (Receiver Operating Characteristic) curves for combining and evaluating datasets and relative performance graphs for viewing datasets relative performance over a suite of tools (16). This chapter covers five known statistics for each dataset in $D$.:

(1) Arithmetic mean. The arithmetic mean of $M$ scores is calculated after the derived statistics are calculated for each dataset in $D$.

(2) Normalized. For each dataset in $D$, normalize the score by subtracting the mean score over all tools then divide by the standard deviation. Combine scores by calculating the arithmetic mean of $M$ normalized scores. Note that this procedure is called standardization in statistics.

(3) Combined. For each dataset in $D$ add $n T P, n F P, n F N, n T N, s T P, s F P$, and $n F N$ as if it were one dataset. Calculate the derived statistical measures over the summed totals.

(4) Relative Performance Graph. Do not combine the $M$ scores. Construct a graph with each dataset along the $X$ axis and relative performance of the $T$ tools along the $Y$ axis. Construct one graph for each derived statistics.

(5) Receiver Operating Characteristic (ROC) Graph. Determine an algorithm parameter $P$. Vary $P$ so that algorithm sensitivity continues to increase while 1 - specificity continues to decrease. The area under the curve (AUC) is an absolute measure of performance, comparable across methods.

Over most derived statistics, Mean, Average, Normalized and Combined summing methods correlate reasonably well on current datasets $(14,17)$. Fig. ??.3 shows the predictions of five different motif prediction methods. Therefore, none of those methods reported false positives on this region. When a tool fails to make predictions over a large number of regions like this, it appears (unfairly) that the tool is specific in locating binding sites because the number of false positives is small. On the other extreme, many tools tend to predict nearly the entire region instead of localizing to the TFBS. Thus, when reading the derived statistics it would 
appear that such methods are sensitive, when they are in fact predicting large contiguous regions of binding sites. For this reason, genome-wide comparisons of binding sites and predicted sites serve as an important sanity check when evaluating statistics.

ROC curves are excellent for comparing the sensitivity/specificity trade-offs of a single parameter (17). ROC curves track the performance of an algorithm over changes over a single parameter. Traditionally, ROC curves have been applied to changing internal algorithm parameters from tight thresholds to more lenient thresholds. This produces a ROC curve that travels straight up and then to the right when the algorithm corresponds exactly to the dataset. Algorithms that poorly represent the problem or problems that are ill-conceived produce a curve will travel straight to the right and then up. Random predictions produce a diagonal line.

We applied ROC analysis to (1) the width of the regulatory regions taken upstream of the gene, (2) the class (protein family) of transcription factor, (3) internal algorithm prediction parameters, and (4) cross-species regulatory region extraction techniques (16). The methods presented here provide insights into the overall trends but some information is lost.

Generalization is difficult since some datasets consist of a few examples, while others have motif instances in the hundreds; some motif instances are highly conserved while others contain a great variability.

\section{Results}

This section presents three illustrative example results of benchmarks that can be generated using a robust database of almost all transcription factor binding sites in the cell and several TFBS prediction methods. Fig. ??.4 illustrates derived statistics summed via the combined method introduced in the previous section. For each transcription factor in RegulonDB, all TFBS were used to create $D=\left\{d_{1}, d_{2}, \ldots\right\}$ where $D$ represents the series of all tests and $d_{1}, d_{2}, \ldots$ each represent a set of co-bound genes, each bound by the same transcription factor. Pipelines were developed for nine different TFBS detection methods and run over $D$ and predictions compared to RegulonDB annotations to determine the core statistics. Each tool was allowed to make three predictions for a single transcription factor. All of the tools in Fig. ??.4 illustrate performance profiles that could be improved. Mitra (18) is an example that, at the thresholds in this example, is sensitive but not specific. Mitra predicts sites over the entire regulatory region. For this reason, it discovers many of the annotated sites, but not because the algorithm is able to find patterns that corresponds to sites. Elph (19), Glam (17) and Gibbs (20) are at the other extreme. These make very few predictions on this dataset, resulting in perceived high specificity. Weeder (21), MotifSampler (22), MEME (23), AlignACE (24), and ANN-Spec (25) all appear to strike a better balance, however nCC remains between 0 and 0.37 for all approaches. There is some possibility that further refinement of tool parameters could yield better performance. Assessments like this provide an overview of where current methods stand and suggest ways of improvements. Fig. ??.5 shows seven motif discovery methods evaluated in an ROC curve. Both single species and cross-species techniques are represented. The benchmark was originally generated using over 20 motif discovery methods but only the top seven tools were plotted for clarity. The ROC curves were generated by running each of the algorithms 10 times; and increasing the amount of predictions each algorithm was allowed to produce (using algorithmic thresholds) after each run. This plot shows some advantages of incorporating cross-species information. Not every tool maintains good prediction accuracy as it is allowed to make additional 
predictions. On this dataset, PhyloGibbs-MP appears to continue to make good predictions as the algorithmic thresholds are lowered.

An example using a relative performance graph is shown on Fig. ??.6. All relative performance graphs contain at least three axes, one for the tools in the study and one for the transcription factor classes in the study, and one for the performance metric. Relative performance graphs are advantageous because they show an in-depth look at relative performance over parts of the dataset for one statistic. The figure shows an in-depth look at $n C C$ over the RegulonDB dataset. Along the $X$ axis is each TFBS evaluated in the assessment. The top graph shows the relative total correlation for all tools in the assessment combined. On the bottom is the relative contribution for each tool for a specific TFBS. Note that columns in the graph have no relationship to one another. TFBS are sorted in this graph by conservation of the sequence at the binding site. This graph illustrates that sequence conservation at the binding site is not enough for accurate TFBS detection.

Fig. ??.6 also demonstrates that no tool in this assessment is clearly dominant in detecting all binding sites of the same transcription factor. Some tools are very good at detecting TFBS for some sites, but not others. Some sites are more easily detected by all tools, and some sites challenge all tools. These results indicate that one tool for detecting all TFBS may not be possible, instead multiple methods for different classes of problems may be more appropriate.

\section{Conclusions}

We introduced an assessment methodology for the performance of TFBS detection algorithms. Platforms such as MTAP make it easier to rank algorithms on multiple criteria and to find effective techniques that solve. We provided a new methodology and tool to compare methods and rank them based on how well they perform on certain subsets of the TFBS detection problem. The key is finding sub-problems of the overall TFBS detection problem that can be solved with reasonable expectation that the algorithm results correspond to real binding sites.

This new methodology is not without problems. First, high quality datasets of TFBS locations need to be standardized and collected in order to use this technology effectively. Second, great care needs to be taken when looking at benchmarking outcomes, as numerical summaries cannot always convey intuition about why certain approaches fail.

We introduced four principal methods for understanding the TFBS detection problem: (1) tabulated results of derived statistics; (2) ROC graphs; (3) sedimentation graphs; and (4) genome-wide prediction visualization. We also introduced a platform, MTAP, for performing these comparisons. MTAP provides the raw data needed to perform comparisons shown in this section. These raw outputs can be customized by the users for diverse interpretations. . A possible application is to compare the pipelines implemented in MTAP with new methods.

When performing assessment, it is important to consider the assumptions of the assessment and ensure that they are in line with the assumptions of the tools being assessed. MTAP was built to assess how well current tools work at automated annotation of genomes. Current tools are expected to perform much better with hand-picked motifs from TFBS databases, although this introduces a certain bias. Assessment must be viewed as a part of the overall system of discovery and verification. It is therefore important that any assessment have a scope consistent with prediction objectives.

\section{Notes \\ 1. Installing MTAP.}


MTAP was developed and tested on Ubuntu Linux. To set up an MTAP run, the user needs to: (1) install motif tool dependencies- this will install tools such as BLAST and MLAGANS that many TFBS tools use as part of their pipelines, (2) install motif discovery tools, (3) format known motif databases, (4) configure MTAP, and (5) run the MTAP analysis. To install MTAP, download the mtap.tar.gz file from http://biobase.ist. unomaha.edu/ mediawiki/index.php/Main_Page and place it in a directory indicated by the \$MTAP_HOME environmental variable. Untar and unzip MTAP to \$MTAP_HOME with the command: tar -xzvf mtap.tar.gz. In this chapter, we will set \$MTAP_HOME=/home. Then the tar command will create the following directory structure:

/home/mtap/pipeline/bin

/home/mtap/pipeline/conf

/home/mtap/pipeline/dumpdir

/home/mtap/pipeline/lib

/home/mtap/pipeline/motifTools

/home/mtap/pipeline/reqs

/home/mtap/pipeline/src

/home/mtap/pipeline/tmp bin contains useful scripts for running MTAP and installing motif tools.

conf contains configuration files for use by scripts in bin.

dumpdir is the location MTAP will place all tool prediction and raw statistics

lib contains libraries needed to run MTAP. Make sure to run compile Java.py before attempting to run MTAP.

motifTools contains TFBS prediction software from other institutions reqs contains libraries and tools for motif prediction tools src contains the MTAP source code tmp is where TFBS databases are placed for MTAP runs and where intermediate results are stored in tool pipelines

\subsection{Install tool dependencies}

Scripts to install tool dependencies exist in /home/mtap/pipeline/bin. First install biopython, bioperl and Java SDK and place them in your path. To install system level dependencies run from bin: . / installprereqs

/home/matp/pipeline/motifTools/Linux-i386/. Cross species regulatory region detection requires RSD. Install RSD with the following command: . /installRSDreqs /home/mtap/pipeline/reqs/RSD-bin/ .

Some motif tools can be distributed with MTAP. For these tools, we provide an automated script for installation. To install run: ./installMotifTools

/home/matp/pipeline/motifTools/Linux-i386/ More information on motif tools is in the next section. MTAP is made aware of the dependencies for your specific architecture through the MTAPglobals.py file found in /home/mtap/pipeline/src/runManager/MTAPglobals.py. Edit MTAPglobals.py to change RUNMANAGERPATH and PROGHOME to:

$$
\text { RUNMANAGERPATH }=\text { "/home/mtap/pipeline/src/runManager" }
$$$$
\text { PROGHOME = "/home/dquest } / \mathrm{mtap} / \text { pipeline". }
$$

MTAPglobals.py also contains many variables to change the MTAP runtime characteristics such as number of predictions allowed, number of sequences required, threshold for site true 
positives and other concepts discussed earlier in the chapter. Edit the following variable lines to make MTAP aware of the local installation:

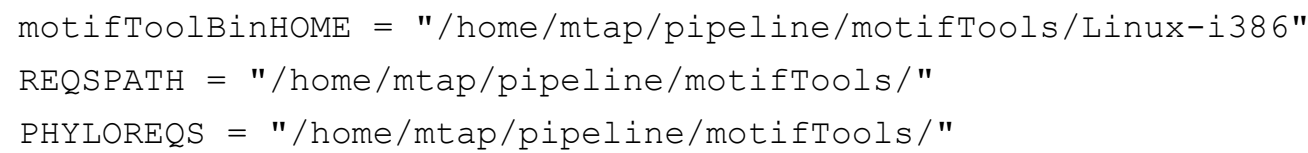

\subsection{Installing TFBS Databases}

To create an MTAP run, one needs to first create the setup files in /tmp. First make a directory for the name of the run. MTAP includes several examples (e.g., /tmp/pito) that can be copied and modified to create new MTAP runs. Following is the directory structure that is needed to create new runs (creating a new run called "Run1"):

The root directory (\$RUN) for the new run.

$\$ R U N / a c c O c$

\$RUN/conf

\$RUN/gbks

\$RUN/ kmraws

\$RUN/motiflists

\$RUN/phylo

\$RUN/protein

$\$ R U N / R S D$

$\$ R U N / x m l s$
Used to hold the accocs.txt association file for relating TFBS location data to Genbank files

Used to backup MTAP configuration files for this specific run.

Holds Genbank files containing genome sequences.

Holds databases for known TFBS binding locations.

Holds the motif.list file containing a unique listing of every transcription factor annotated in the kmraw database.

Holds the Phylo.txt file for relating the .gbk files and for storing 16sRNA phylogenetic trees and multiple sequence alignments.

Holds translated .faa files for each coding sequence in the .gbk file and blast databases for searching.

Holds ortholog tables for cross species comparisons

Location to store xml configuration files used by java components

Once the directory structure is made, the MTAP user need only copy Genbank files into the gbks directory, copy tab-delimited TFBS data into the kmraw directory, create the phylo.txt file and create the accocs. txt file. MTAP automatically creates the rest of the needed information for the run. Then the MTAP user should change the settings for the MTAP run in MTAPglobals.py and MTAPdbSetup.py to ensure MTAP will run correctly. At this time, they are ready to run MTAP (python /home/bin/MTAP.py). Examples of known motif databases acceptable by MTAP and Genbank files are in the MTAP.tar.gz download for reference.

\section{Running Motif Detection Tools.}


Motif tools are as variable as the people that develop them. Each takes a multi-Fasta file representing multiple regulatory regions. That is where the similarities end. Each tool produces a specifically formatted output file and takes a specific array of inputs. MTAP unifies all tools by converting each arbitrary output into a unified format (.gff) that represents predicted features from each tool in the dataset. MTAP also produces each arbitrary input needed by the program. When MTAP is run, it creates a run database consisting of all run tests in the database. This database consists of run tuples of the form:

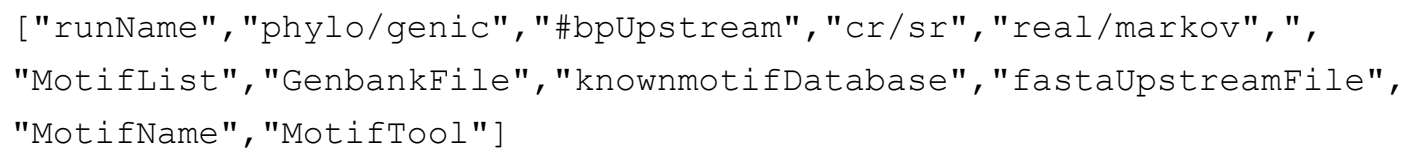

These run tuples are constructed dynamically by permuting all options available in the src/runManager/MTAPdbSetup.py configuration file and from the data found in the TFBS database in /tmp (see previous section). Each MTAP run consists of all possible permutations of the variables found in MTAPdbSetup.py file. These permutations are translated into jobs. The collection of all MotifTool pipeline jobs in a single MTAP run is logged in a file called RUNFILE. RUNFILE exists in / home/dumpdir/runName/RUNFILE. The directory structure under /home/dumpdir/ corresponds to the tuples in the RUNFILE. For example, consider a run using Weeder to find binding sites for the CRP transcription factor in E. coli K12 (NC_000913.gbk). Assume RegulonDB is the dataset we wish to use for evaluation, and that we want to take $400 \mathrm{bp}$ upstream of every gene regulated by CRP as annotated in RegulonDB. The MotifList file for all unique motifs in RegulonDB is called: v2008_NC_000913. Assuming we call the run "Run1", the tuple for this job will look like: ["Run1", "genic", "400", "cr", "real", "v2008_NC_000913", "knownmotifs.regulondb.v2008", "NC_0 The job tuple indicates the location where the tool will be run on the local file structure. The above example will be run in \$RUNDIR =/home/mtap/pipeline/dumpdir/Run1/genic/ $400 / \mathrm{cr} / \mathrm{real} /$. Unified . gff files for plotting in tools such as gbrowse are available in \$RUNDIR/gff. The raw statistics files for analysis are available in \$RUNDIR/stats . Specific tool thresholds and pipelines can be modified by changing the tool driver scripts found in /home/mtap/pipeline/src/runManager/motifTools. Sophisticated data collection scripts for analyzing gene regulatory networks with graph theory and for plotting changes across run parameters are available in src/runManager.

\section{Figure Legends}

Fig. ??.1. Evaluating TFBS discovery algorithms. (A) First, all known regulatory regions from a genome are assembled into a database. We then apply a reduction function, $t$, over all regulatory elements to determine a set of co-bound regulatory sequences (a). Function $t$ uses evidence from ChIP-chip, ChIP-seq, or a TFBS database to include only regions bound by transcription factor $i$. The result of this pruning is shown in $B$. This results in $n$ subsets $B_{1}, B_{2}, \ldots, B_{n}$ one for each transcription factor. For each regulatory subset $\left(B_{i}\right)$ we apply additional functions, $h_{l}, h_{2}, \ldots, h_{n}$ to collect background sequence data, to collect the orthologous regulatory regions in other genomes. These sequences are then fed into the prediction pipeline $(D)$, which calculates the background probability of a pattern in the sequences in $A$ and from any other sequences collected in $C$. The pipeline then generates a set of predictions corresponding to possible binding sites. Prediction positions are marked in a standard format shown in $E$. 
Fig. ??.2. The seven core statistics collected to assess the accuracy of TFBS detection tools.

Fig. ??.3. An example regulatory region 400 -bp upstream of $f e p B$ and downstream ent $C$ in the Escherichia coli K12 genome. Features are mapped on the extracted upstream regulatory region, shown in black. From top to bottom features are mapped to the region as follows: (1) promoters in the database, (2) known TFBS from RegulonDB. Predictions from five TFBS prediction algorithms are marked below those from the experimentally determined TFBS from the database: Weeder (3), AlignACE (4), MEME (5), Glam (6) and Gibbs (7). Two promoters (sigma factor binding sites) lie within the regulatory region (shown by dashed lines) containing binding sites for transcription factors $C R P$ and Fur. Experimentally determined $C R P$ positions are furthest to the right and seventh from the left. Fur sites occupies the rest of the verified binding positions. Collectively, the five TFBS prediction methods cover nearly the entire region, a typical outcome in current prediction technology. Some tools like Weeder do not make predictions for both TFBS classes.

Fig. ??.4. Derived statistics for nine regulatory motif detection methods.

Fig. ??.5. PhyloMEME (a version of MEME run on regulatory regions from multiple species), PhyME, PhyloGibbs-MP, Phylo-Weeder, ANN-Spec, Motifsampler, and Mitra represent state of the art motif detection algorithms. This composite ROC curve shows a side-by-side comparison of phylogenetic-assisted and purely sequence-based tools. At the nucleotide level, performance is virtually random. At the site level, phylogenetics-based tools such as PhyloGibbs-MP outperform single-genome methods. However, several methods perform hardly better than random. This result is expected given the performance of several methods shown in Fig. ??.4. Tools cover large portions of the regulatory region with predictions, many of them overlapping known binding sites.

Fig. ??.6. $n C C$ was calculated over RegulonDB by considering the top 3 predictions from each tool. $n C C$ over 16 tools assessed in this study represented in a heatmap. $n C C$ values range from 0 (black) to 0.37 (white). Binding sites are sorted from high information content at the conserved site (bottom) to low information content (top). $n C C$ values do not increase as conservation at the site increases, most likely due to competing background signals in the upstream regulatory regions. In this table, $n C C$ is negatively impacted for ELPH, Gibbs, Glam, PhyloMEME, PhyloGibbs, PhyloGibbsMP, and PhyME because the number of predictions is low (easily overcome for some tools by considering more sites). Binding sites for some TFs such as AgaR are relatively accurately predicted by many tools. Other TFs such as EvgA pose a greater challenge. JAMM-b is a Bayesian filter for combining multiple methods. JAMM-i is the same filter, with a length based constraint relative to the promoter. No tool is clearly dominant for every transcription factor. 
Tables

Statistic

$n T P$

\section{Definition}

$n F N$

$u_{i, j}$ is both annotated and predicted.

$n F P$

$u_{i, j}$ is annotated but not predicted.

$n T N$

$u_{i, j}$ is predicted but not annotated.

$u_{i, j}$ is neither annotated nor predicted

Table ??.1. Nucleotide Level Statistics. $u_{i, j}$ is the upstream regulatory sequence $j$ at position $i$.

\section{Statistic}

sTP

$s F N$

$s F P$

$s T N$

\section{Definition}

Number of known sites overlapped by predicted sites.

Number of known sites not overlapped by predicted sites.

Number of predicted sites not overlapped by known sites.

$$
s T N=\frac{n T P+n F P+n F N+n T N}{N u m b e r S e q u e n c e s}-\mathbf{s T P}-\mathbf{s F N}-\mathbf{s F P}
$$

Table ??.2. Site Level Statistics.

Sensitivity:

Specificity:

PositivePredictiveValue:

Matthews Correlation

Coefficient

Correlation Coefficient:

Site level average site performance:

Table ??.3. Statistics for Evaluating Motif Prediction Algorithm Implementations.

\section{References}

1. Das, M., and Dai, H. (2007) A survey of DNA motif finding algorithms. BMC Bioinformatics 8, 1-13 
2. Davidson, E.H., Rast, J.P., Oliveri, P., et al. (2002) A genomic regulatory network for development. Science 295, 1669-1678.

3. Stathopoulos, A., and Levine, M. (2005) Genomic regulatory networks and animal development. Dev Cell 9, 449-462.

4. Imai, K., Levine, M., Satoh, N., et al. (2006) Regulatory Blueprint for a Chordate Embryo. Science 312, 1183-1187.

5. Salgado, H., Santos-Zavaleta, A., Gama-Castro, S., et al. (2006) The comprehensive updated regulatory network of Escherichia coli K-12. BMC Bioinformatics 7, 1-5.

6. Shen-Orr, S.S., Milo, R., Mangan, S., et al. (2002) Network motifs in the transcriptional regulation network of Escherichia coli. Nature Genet 31, 64 - 68.

7. Salgado, H., Gama-Castro, S., Martaenez-Antonio, A., et al. (2004)

RegulonDB (version 4.0): transcriptional regulation, operon organization and growth conditions in Escherichia coli K-12. Nucleic Acids Res 32.

8. Karp, P., Riley, M., Saier, M., et al. (2002) The EcoCyc Database. Nucleic Acids Res 30, 56-58.

9. Wingender, E., Dietze, P., Karas, H., et al. (1996) TRANSFAC: a database on transcription factors and their DNA binding sites. Nucleic Acids Res 24, 238-241.

10. Ishii, T., Yoshida, K.-I., Terai, G., et al. (2001) DBTBS: a database of Bacillus subtilis promoters and transcription factors. Nucleic Acids Res 29, 278-280.

11. Kazakov, A.E., Cipriano, M.J., Novichkov, P.S., et al. (2006)

RegTransBase--a database of regulatory sequences and interactions in a wide range of prokaryotic genomes. Nucleic Acids Res. 35 (Database Issue): D407-D412.

12. Maench, R., Hiller, K., Barg, H., et al. (2003) PRODORIC: prokaryotic database of gene regulation. Nucleic Acids Res 31, 266-269.

13. Meng, H., Banerjee, A., and Zhou, L. (2006) BLISS: binding site level identification of shared signal-modules in DNA regulatory sequences. $B M C$

Bioinformatics 7, 287.

14. Tompa, M., Li, N., Bailey, T., et al. (2005) Assessing computational tools for the discovery of transcription factor binding sites. Nature Biotechn 23, 137-144. 15. Sandve, G., Abul, O., Walseng, V., et al. (2007) Improved benchmarks for computational motif discovery. BMC Bioinformatics 8, 193.

16. Quest, D. (2009).

17. Frith, M.C., Hansen, U., Spouge, J.L., et al. (2004) Finding functional sequence elements by multiple local alignment. Nucleic Acids Res 32, 189-200.

18. Eskin, E., and Pevzner, P.A. (2002) Finding composite regulatory patterns in DNA sequences. Bioinformatics 18 Suppl 1 pS354-S363.

19. (2000) ELPH Manual from the Center for Bioinformatics and Computational Biology.

20. Lawrence, C.E., Altschul, S.F., Boguski, M.S., et al. (1993) Detecting subtle sequence signals: a Gibbs sampling strategy for multiple alignment. Science 262, 208-214.

21. Pavesi, G., Mereghetti, P., Mauri, G., et al. (2004) Weeder Web: discovery of transcription factor binding sites in a set of sequences from co-regulated genes. Nucleic Acids Res 32.

22. Thijs, G., Lescot, M., Marchal, K., et al. (2001) A higher-order background model improves the detection of promoter regulatory elements by Gibbs sampling. Bioinformatics 17, 1113-1122. 
23. Bailey, T.L., and Elkan, C. (1994) Fitting a mixture model by expectation maximization to discover motifs in biopolymers. Proceedings / International Conference on Intelligent Systems for Molecular Biology ; ISMB International Conference on Intelligent Systems for Molecular Biology 2, 28-36.

24. Hughes, J.D., Estep, P.W., Tavazoie, S., et al. (2000) Computational identification of cis-regulatory elements associated with groups of functionally related genes in Saccharomyces cerevisiae. J Mol Biol 296, 1205-1214.

25. Workman, C.T., and Stormo, G.D. (2000) ANN-Spec: a method for discovering transcription factor binding sites with improved specificity. Pac Symp Biocomput, 5: 467-478. 